THE JOURNAL OF INFECTIOUS DISEASES - VOL. 146, NO. 2 - AUGUST 1982

(C) 1982 by The University of Chicago. All rights reserved, $0022-1899 / 82 / 4602-0012 \$ 00.75$

\title{
Protection Against Chronic Pyelonephritis in Rats by Suppression of Acute Suppuration: Effect of Colchicine and Neutropenia
}

\author{
Jacques Bille and Michel P. Glauser
}

\begin{abstract}
From the Division des Maladies Infectieuses, Department de Médicine, Centre Hospitalier Universitaire Vaudois, Lausanne, Switzerland
\end{abstract}

\begin{abstract}
Previous experiments in rats have suggested that renal scarring after acute, obstructive pyelonephritis due to Escherichia coli results from parenchymal damage due to acute inflammation and suppuration. To assess the role of acute infiltration by polymorphonuclear leukocytes (PMNLs) in the pathogenesis of chronic pyelonephritis (CPN), rats were either treated with colchicine to depress leukcoyte motility or rendered neutropenic with a single dose of cyclophosphamide. Colchicine given during acute pyelonephritis reduced kidney inflammation and protected against CPN two months later. Similarly, neutropenia reduced acute inflammation and protected against chronic parenchymal destruction and scarring. Protection against renal scarring in both colchicine-treated and neutropenic rats occurred despite higher renal bacterial counts during acute pyelonephritis. These experiments provide further evidence that CPN (renal scarring) results from kidney damage that occurs during early acute obstructive pyelonephritis. This damage appears to result from infiltration of the kidney by PMNLs rather than direct damage from bacterial infection.
\end{abstract}

The mechanisms that lead to the development of chronic pyelonephritis (CPN) after acute renal infection are not well defined. Persistent infection does not seem to play a critical role in the development of renal scarring, because kidneys from patients with CPN are often sterile at autopsy $[1,2]$. In experimental pyelonephritis, viable bacteria are not required for progression of the lesions [3-5]. Furthermore, bacterial antigens persisting in the late phase of human and experimental pyelonephritis $[2,6,7]$ have been shown not to trigger significant kidney destruction $[6,7]$. Two other immune mechanisms have been proposed. Either alterations in antigenicity of kidney tissue [8] or common antigens which are shared by Escherichia coli and renal tissue [9] have been postulated to

Received for publication July 20,1981 , and in revised form March 3, 1982.

Portions of this work appeared as an abstract (no. 532) in the Proceedings of the 21st Interscience Conference on Antimicrobial Agents and Chemotherapy and in Clinical Research 28:364A, 1980.

This work was supported in part by grant no. 3.814-0.79 from the Swiss Foundation for Scientific Research.

We thank Dr. J. A. McCutchan for assistance in preparation of the manuscript and S. Bovey for secretarial assistance.

Please address requests for reprints to Dr. Michel P. Glauser, Division des Maladies Infectieuses, Départment de Médicine, Centre Hospitalier Universitaire Vaudois, 1011 Lausanne, Switzerland. stimulate a chronic, destructive immune response. However, the demonstration of antibodies to kidney tissue in CPN has been unsuccessful so far, both in humans [10] and in experimental animals [11]. Thymus-dependent autoimmune phenomena have been shown not to play a significant role in the development of CPN in rats [5, 12]. In fact, athymic, nude mice challenge iv with Streptococcus faecalis seem to develop more intense kidney infection and scarring than their normal littermates [13].

To investigate the mechanisms that lead to CPN, we have used a model of ascending obstructive pyelonephritis due to $E$. coli that resembles severe human kidney infection because the disease is acquired by the retrograde route in the presence of an obstruction [14]. Previous experiments in which rats were treated with antibiotics starting at different times during acute pyelonephritis have suggested that the acute inflammatory process plays a major role in the development of kidney scars leading to CPN and that infection seems less important [5]. In those studies, however, the diminished inflammation by early treatment might have been due to bacterial inhibition by antibiotics. During inflammatory processes, polymorphonuclear leukocytes (PMNLs) can damage tissue by exocytosis of lysosomal enzymes. Therefore, we gave rats with acute pyelonephritis 
either colchicine, thus impairing PMNL movement, or cyclophosphamide, thus inducing severe neutropenia.

\section{Materials and Methods}

Induction of pyelonephritis. Retrograde pyelonephritis was induced in male Wistar rats weighing 200-250 g (Madörin, Füllingsdorf, Switzerland) [14]. In brief, $10^{3} \mathrm{E}$. coli type 06 organisms (Williams strain) [14] were slowly infused into the bladder and a ligature was loosely tied around the left ureter; $18 \mathrm{hr}$ later the ligature was removed. This procedure produces partial obstruction followed by severe unilateral pyelonephritis. Three days after this operation, the pyelonephritic kidneys are greatly enlarged and display numerous abcesses over the cortex [14]. Two months after the operation, the kidneys have extended scars with severe destruction of kidney parenchyma [14].

Experimental design and killing of rats. In this model of pyelonephritis, inflammation, as measured by the ratio of the weight of the left pyelonephritic kidney to that of the right normal kidney, reaches a peak three days after the operation and bacterial inoculation [5]. Colchicine-treated, neutropenic, and control rats were killed at this time in order to evaluate the effect of colchicine and neutropenia on acute pyelonephritis. The kidneys were examined for gross evidence of acute pyelonephritis after three days or for scars of CPN after two months. The distribution of abscesses or scars on the surface of the kidney was graded by dividing the kidney into eight equal parts and counting the number of parts where there was at least one abscess or scar. A distribution index was calculated from the sum of the number of parts having abscesses or scars [14]. The kidneys were weighed and cut in half; one half was homogenized in $0.85 \% \mathrm{NaCl}$ and serially diluted before plating onto MacConkey's agar (Difco Laboratories, Detroit) for colony counts expressed in $\log \mathrm{cfu}$. The other half of each kidney was processed for histologic examination.

Severity of pyelonephritis. Kidney weight provides the best quantitative measure of the severity of pyelonephritis-it increases in proportion to suppuration during acute obstructive pyelonephritis, whereas it decreases in proportion to the destruction of kidney tissue during CPN [15]. To minimize the effects of variation of kidney weights among animals, the ratio of the left kidney weight to the right kidney weight (the $\mathrm{L}: \mathrm{R}$ weight ratio) was used [5]. This is especially important when dealing with long-term experiments, wherein the total body weight may vary greatly among animals. The $L: R$ weight ratio also expresses the compensatory hypertrophy of the right kidney during CPN, which is proportional to the destruction and shrinkage of the left kidney [15].

Administration of colchicine. Colchicine powder (Fluka, Buochs, Switzerland) was diluted in sterile water to a concentration of $1 \mathrm{mg} / \mathrm{ml}$. Colchicine was administered ip daily to the rats in a dosage of $0.4 \mathrm{mg} / \mathrm{kg}$ of body weight per day for three consecutive days. The first injection was given one day before the operation. Control rats were given one injection of $0.1 \mathrm{ml}$ of $0.85 \% \mathrm{NaCl}$ ip daily for three consecutive days.

Administration of cyclophosphamide and induction of neutropenia. Cyclophosphamide powder (Endoxan; Asta Werke, Bielefeld, Federal Republic of Germany) was diluted in sterile water to a concentration of $25 \mathrm{mg} / \mathrm{ml}$. Cyclophosphamide was given ip to the rats four days before the operation in a dose of $100 \mathrm{mg} / \mathrm{kg}$ of body weight. Control rats were given $1.0 \mathrm{ml}$ of $0.85 \% \mathrm{NaCl}$ ip. Leukocyte counts of the tail blood were made with a hemacytometer.

Statistical evaluation. The number of $\mathrm{cfu} / \mathrm{g}$ of kidney, the $L: R$ weight ratio of the kidneys, and the surface area of the kidney occupied by abscesses were compared by Student's unpaired $t$-test.

\section{Results}

Effect of colchicine treatment on pyelonephritis. Colchicine treatment was associated with early mortality in the rats receiving the drug. During the first three days after the operation, seven $(14 \%)$ of 49 colchicine-treated rats died, four in the acute and three in the chronic pyelonephritis experiments. No deaths occurred in the control rats. The severity of the disease in the dead animals as measured by the $L: R$ weight ratio, the distribution of macroscopic lesions, and the bacterial counts were not different from those observed in 
Table 1. Effect of treatment with colchicine on the severity of experimentally induced pyelonephritis in rats.

\begin{tabular}{|c|c|c|c|c|c|c|}
\hline \multirow[b]{2}{*}{ Laboratory results } & \multicolumn{3}{|c|}{ Acute phase (three days after induction) } & \multicolumn{3}{|c|}{ Chronic phase (two months after induction) } \\
\hline & Control $(n=14)$ & $\begin{array}{c}\text { Colchicine-treated } \\
(n=18)\end{array}$ & $P$ & Control $(n=18)$ & $\begin{array}{l}\text { Colchicine-treated } \\
\quad(n=24)\end{array}$ & $P$ \\
\hline $\mathbf{L}: \mathbf{R}$ weight ratio & $1.77 \pm 0.3$ & $1.56 \pm 0.2$ & 0.02 & $0.35 \pm 0.1$ & $0.48 \pm 0.2$ & 0.02 \\
\hline Distribution index & $5.3 \pm 2.1$ & $6.5 \pm 1.4$ & NS & $6.61 \pm 1.4$ & $5.50 \pm 2.2$ & NS \\
\hline Bacterial count (log $\mathrm{cfu} / \mathrm{g})$ & $7.60 \pm 1.2$ & $8.40 \pm 0.8$ & 0.031 & $3.84 \pm 2.3$ & $3.19 \pm 2.5$ & NS \\
\hline
\end{tabular}

NOTE. Colchicine $(0.4 \mathrm{mg} / \mathrm{kg}$ of body weight per day) was administered ip to rats for three days beginning one day before pyelonephritis was induced in the left kidney by operation [14]. Control rats were given one ip injection of $0.1 \mathrm{ml} \mathrm{of} 0.85 \% \mathrm{NaCl}$ per day for three days. Three days after the operation, the ratio of the weight of the left kidney to the weight of the right kidney (L: $R$ weight ratio) was calculated to measure the intensity of the inflammation of the left kidney. Two months later, the $L: R$ weight ratio was calculated to express the destruction of tissue in the left kidney. The surface of the left kidney was divided into eight equal sections and the distribution index was defined as the number of sections with one or more abscesses or scars. Infection was expressed as the log of the number of cfu of Escherichia coli type 06/g of left kidney homogenate. Data are means $\pm \mathrm{SD}$. NS $=$ not significant.

colchicine-treated rats that were killed at the same time.

Table 1 compares pyelonephritis in colchicinetreated rats and control rats. When expressed by the $L: R$ weight ratio, acute inflammation was slightly but significantly reduced in colchicinetreated rats. The distribution of lesions on the surface of the left kidney in the colchicine-treated rats was not different from that in the control rats, but the gross abscesses were smaller. In contrast to the reduction of $L: R$ weight ratio, the bacterial counts in the kidneys were significantly higher in colchicine-treated rats than in the control rats.

Table 1 also shows the long-term effect of colchicine treatment. There was significant protection of the colchicine-treated rats against the parenchymal destruction of the pyelonephritic kidney compared with the control animals. Despite a similar distribution of scars at the surface of the kidney, scars were smaller and less numer- ous in colchicine-treated rats. Bacterial counts were similar in both groups of rats after two months.

Effect of neutropenia on pyelonephritis. Administration of cyclophosphamide four days before the operation resulted in severe leukopenia and neutropenia at the time of the operation and bacterial inoculation. The mean \pm sD leukocyte count in the peripheral blood was reduced from $9,300 \pm 2,800$ cells $/ \mathrm{mm}^{3}$ in the control rats to $1,010 \pm 360$ cells $/ \mathrm{mm}^{3}$ on the day of the operation, to $820 \pm 380$ cells $/ \mathrm{mm}^{3}$ the following day (coinciding with the end of the ureteral obstruction), and to $1,780 \pm 930$ cells $/ \mathrm{mm}^{3}$ two days after the operation. No circulating PMNLs were found on the first two days after the operation.

Cyclophosphamide treatment was accompanied by $22 \%$ mortality (five of 25 rats) during the first three days after the operation. No deaths occurred in the control rats. Pyelonephritis in the dead animals as measured by the $L: R$ weight ratio of the

Table 2. Effect of treatment with cyclophosphamide on the severity of experimentally induced pyelonephritis in rats.

\begin{tabular}{|c|c|c|c|c|c|c|}
\hline \multirow[b]{2}{*}{ Laboratory results } & \multicolumn{3}{|c|}{ Acute phase (three days after induction) } & \multicolumn{3}{|c|}{ Chronic phase (two months after induction) } \\
\hline & Control $(n=21)$ & $\begin{array}{l}\text { Cyclophosphamide- } \\
\text { treated }(n=9)\end{array}$ & $P$ & Control $(n=24)$ & $\begin{array}{l}\text { Cyclophosphamide- } \\
\text { treated }(n=10)\end{array}$ & $P$ \\
\hline L:R weight ratio & $2.03 \pm 0.3$ & $1.63 \pm 0.3$ & 0.0023 & $0.31 \pm 0.15$ & $0.50 \pm 0.2$ & 0.0085 \\
\hline Distribution index & $5.5 \pm 2.1$ & $6.05 \pm 1.6$ & NS & $6.64 \pm 2.0$ & $6.96 \pm 1.4$ & NS \\
\hline Bacterial count $(\log \mathrm{cfu} / \mathrm{g})$ & $8.29 \pm 1.0$ & $9.45 \pm 1.1$ & 0.086 & $3.43 \pm 2.0$ & $4.26 \pm 2.3$ & NS \\
\hline
\end{tabular}

NOTE. Cyclophosphamide ( $100 \mathrm{mg} / \mathrm{kg}$ of body weight) was administered ip to rats four days before pyelonephritis was induced in the left kidney by operation [14]. Control rats were given $1.0 \mathrm{ml}$ of $0.85 \% \mathrm{NaCl}$ ip at the same time. Three days after the operation, the ratio of the weight of the left kidney to the weight of the right kidney ( $L: R$ weight ratio) was calculated to measure the intensity of the inflammation of the left kidney. Two months later, the $L: R$ weight ratio was calculated to express the destruction of tissue in the left kidney. The surface of the left kidney was divided into eight equal sections, and the distribution index was defined as the number of sections with one or more abscesses or scars. Infection was expressed as the log of the number of cfu of Escherichia coli type $06 / \mathrm{g}$ of left kidney homogenate, Data are means $\pm \mathrm{SD}$. NS $=$ not significant. 
kidneys, the distribution of macroscopic lesions, and the bacterial counts was not different from that in surviving neutropenic rats killed at the same time. Table 2 compares pyelonephritis in neutropenic and control rats. When expressed by the $L: R$ weight ratio, inflammation during the acute phase of the disease was reduced in the cyclophosphamide-treated rats compared with that in control rats. In contrast, the bacterial counts were significantly higher.

As in the colchicine experiments, the distribution of abscesses over the renal cortical surface was similar in neutropenic and control rats, but the size and the number of abscesses was markedly reduced. This striking difference is shown in figure 1.

After two months of infection, cyclophosphamide-treated rats displayed a significant reduction in parenchymal destruction and scarring, despite a slightly higher number of residual bacteria. Furthermore, although the distribution of scars was similar to that in control rats, the scars were smaller and fewer.

Microscopic examinations of sections of acutely infected kidneys showed dramatic differences between neutropenic rats and control rats. Control rats had many large abscesses containing innumerable PMNLs. Single abscesses extended from the medulla through the cortex and invariably distended the overlying capsule. Lesions in neutropenic animals which looked macroscopically like abscesses, appeared microscopically to contain masses of bacteria within distended tubules, but contained only a few PMNLs. These lesions were smaller and seldom distended the overlying capsule. Thus, lesions in acute pyelonephritis in neutropenic rats were both fewer and quite different in microscopic appearance from those in control rats. These histologic differences probably accounted for the differences in kidney weight.

Despite differences in kidney weight during the acute phase, the histology of the scars examined two months after the operation was similar in cyclophosphamide-treated and control rats.

\section{Discussion}

Previous studies of the pathogenesis of CPN after acute, ascending, obstructive pyelonephritis in the rat [5] have suggested that acute inflammation and suppuration determined the severity of residual
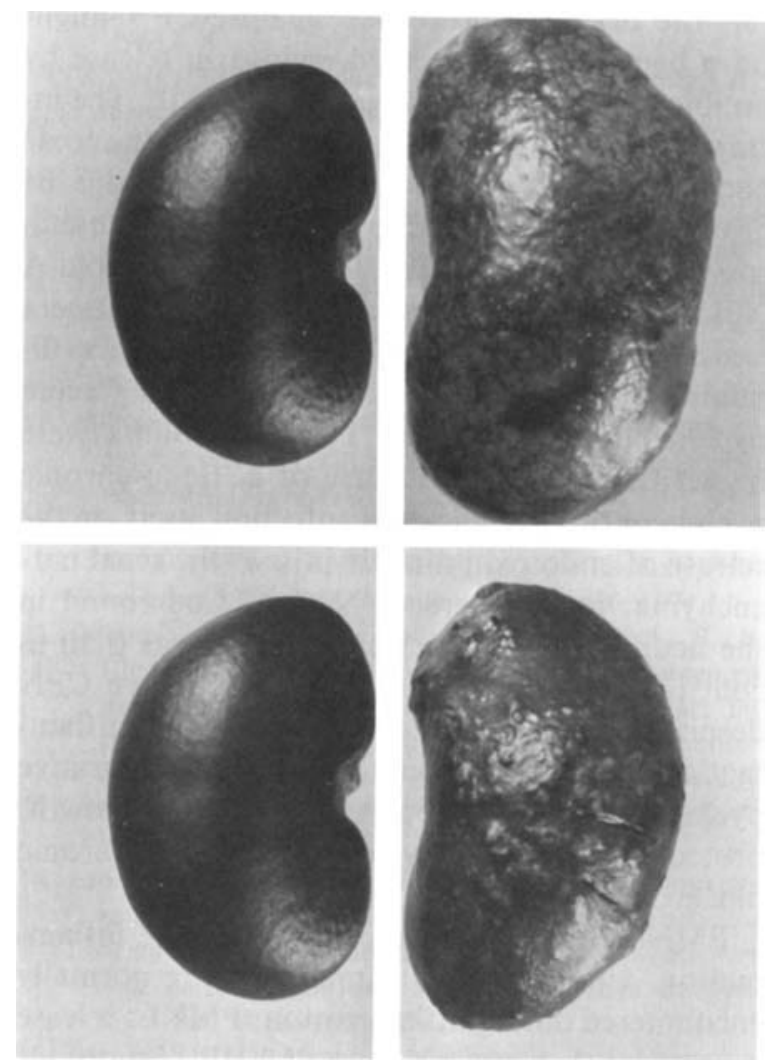

Figure 1. Control (left) and acutely pyelonephritic (right) kidneys from (top) untreated and (bottom) neutropenic rats. Cyclophosphamide was given to rats four days before the induction of pyelonephritis to induce severe leukopenia and neutropenia.

renal scarring. If the peak of inflammation was prevented by early antibiotic therapy, very little parenchymal destruction occurred. Even if low doses of antibiotic sufficient to suppress acute inflammation but not to sterilize the kidney were given, little scarring developed despite persistent infection throughout the course of the disease. Moreover, if antibiotic treatment was started after the peak inflammatory process had fully developed, severe renal destruction and CPN evolved in the absence of bacteria. These two observations suggest that the bacteria themselves act only to incite the acute inflammatory response and thereafter play an insignificant role in the development of CPN. Similar observations stressing the need for early antibiotic treatment to prevent renal scars have been recently made by other investigators [16].

In the antibiotic experiments mentioned above 
[5], the dramatic protection against CPN might have been due to decreased endotoxin release by antibiotic suppression of bacterial growth. The intrarenal injection of dogs with lipid $\mathrm{A}$, the toxic component of lipopolysaccharide endotoxins of gram-negative bacteria, produces an inflammatory response that is related to the dose of lipid A [17]. In the present report, colchicine treatment and cyclophosphamide-induced neutropenia diminished the peak of inflammation during acute pyelonephritis, but renal bacterial counts were higher throughout the course of acute-to-chronic pyelonephritis. If bacterial infection itself or the release of endotoxin directly injures the renal parenchyma, more severe CPN should be found in the neutropenic or colchicine-treated rats than in control rats. Instead, we found less severe CPN despite higher bacterial counts. Decreased inflammation during the acute phase of suppurative pyelonephritis, not depressed bacterial growth, protected against kidney destruction in the chronic phase of the disease.

PMNLs are a crucial element in acute inflammation. Under various stimuli that are normally encountered during inflammation, PMNLs release substances that injure tissues. [18, 19]. Studies of acute myocardial infarction in rats have shown that tissue damage is directly related to PMNL infiltration and is reduced by measures which inhibit exudation [20]. In gouty arthritis, inflammation is mainly mediated by the release of inflammatory substances from PMNLs actively phagocytosing urate crystals [21]. Colchicine has been used for centuries to treat this condition. Its anti-inflammatory effect has been shown to be due to interference with leukocyte locomotion and chemotaxis and to diminished lysosomal degranulation [22]. In experimental pyogenic infection [23], colchicine has been shown to delay the delivery of PMNLs to the site of infection and thus reduce inflammation. However, infection was more severe in these experiments, at least in part because of the delay in delivery of leukocytes. Colchicine acted similarly in acute pyelonephritis in rats in the present study. The inflammatory processes as measured by the $L: R$ weight ratios of the kidneys were reduced after colchicine treatment, whereas bacterial counts increased. This resulted in reduced parenchymal destruction in the chronic phase, suggesting that the toxic and tissue-destructive processes during acute inflammation could be in part due to infiltration of leukocytes that was more important than infection in the pathogenesis of renal scars.

Whereas colchicine treatment provided indirect support for a crucial role of PMNLs in the pathogenesis of renal scarring, experiments in rats rendered neutropenic during acute pyelonephritis provided more direct evidence. It has been shown in another model of acute pyelonephritis that complement depletion by means of cobra venom factor results in diminished exudation of PMNLs in the renal parenchyma and preserves the morphology of the kidney [24]. More recently, Shimamura [25] observed a direct relationship between infiltration of PMNLs and renal tissue damage during the first two days of renal infection in neutropenic rats. In our neutropenic rats with acute pyelonephritis, infection in the kidneys was as widespread as in the control rats but was less severe as judged by smaller abscess size, lower kidney weight, and much less infiltration of PMNLs. In addition, renal scarring two months later was diminished.

Because treatments with colchicine and cyclophosphamide were accompanied by some mortality, we explored the possibility that both drugs could influence food and water intake. We wondered if the reduced inflammation found during acute pyelonephritis could have been due to dehydration resulting from diminished water intake. This was not the case, because pyelonephritic rats housed in metabolic cages and given the same amount of food and water as taken by cyclophosphamide-treated rats had as severe pyelonephritis as control rats fed as much as they wanted.

Experiments by Miller et al. [26] using cyclophosphamide to treat pyelonephritis in rats illustrates the role of early infiltration by PMNLs in the pathogenesis of renal scars. In these experiments, cyclophosphamide was administered two and four days after initiating renal infection. The resulting neutropenia, which was delayed in relation to the peak of inflammation, did not prevent renal scars. The contrast between our results and those with delayed induction of neutropenia support the view that initial infiltration by PMNLs produces the renal injury that ultimately results in CPN.

Cyclophosphamide might prevent renal scars by suppression of humoral or cellular immune mechanisms responsible for renal parenchymal dam- 
age. Indeed, next to its immediate leukopenic effect, cyclophosphamide has a profound effect on both $T$ and B lymphocytes [27]. However, extensive investigation has not shown a role for humoral immunity in the pathogenesis of CPN $[2,5-7$, $10,11]$. In the present report, $E$. coli agglutinin levels measured two months after infection were similar in cyclophosphamide-treated and in control pyelonephritic rats (mean \pm SD $\log _{2}$ serum titer, $19.8 \pm 1.8$ and $20.2 \pm 2.4$, respectively). Thus, it seems unlikely that parenchymal damage was prevented by diminished antibody production. Cellular immune mechanisms have been shown not to be responsible for kidney damage during renal infection [12, 28]. Furthermore, in our model, rats thymectomized at birth showed no difference in the extent of CPN from that observed in sham-thymectomized rats [5]. If cyclophosphamide given just before infection in our experiments had acted by preventing a cellular immune mechanism, the administration of the drug just after initiation of infection as done by Miller et al. [26] should have also diminished renal scars. Because this was not the case, it reinforces our interpretation that PMNLs, rather than cellular or humoral immunity, mediate tissue damage during the inflammatory processes resulting from infection in the kidney.

\section{References}

1. Angell, M. E., Relman, A. S., Robbins, S. L. "Active" chronic pyelonephritis without evidence of bacterial infection. N. Engl. J Med. 2781303-1308, 1968.

2. Aoki, S., Imamura, S., Aoki, M., McCabe, W. R. "Abacterial" and bacterial pyelonephritis: immunofluorescent localization of bacterial antigen. N. Engl. J. Med. 281: 1375-1382, 1969.

3. Kalmanson, G. M., Sommers, S. C., Guze, L. B. Pyelonephritis. VII. Experimental ascending infection with progression of lesions in the absence of bacteria. Archives of Pathology 80:509-516, 1965.

4. Glassock, R. J., Kalmanson, G. M., Guze, L. B. Pyelonephritis. XVIII. Effect of treatment on the pathology of enterococcal pyelonephritis in the rat. Am. J, Pathol. 76:49-60, 1974.

5. Glauser, M. P., Lyons, J. M., Braude, A. I. Prevention of chronic experimental pyelonephritis by suppression of acute suppuration. J. Clin. Invest. 61:403-407, 1978.

6. Sanford, J. P., Hunter, B. W., Donaldson, P. Localization and fate of Escherichia coli in hematogenous pyelonephritis. J. Exp. Med. 116:285-294, 1962.

7. Cotran, R. S. Retrograde Proteus pyelonephritis in rats: localization of antigen and antibody in treated sterile pyelonephritic kidneys. J. Exp. Med. 117:813-821, 1963.

8. Kováts, T. G. The role of endotoxin in autoimmune processes. Naturwissenschaften 48:572-573, 1961.

9. Holmgren, J., Hanson, L. A., Holm, S. E., Kaijser, B. An antigenic relationship between kidney and certain $E s$ cherichia coli strains. Int. Arch. Allergy Appl. Immunol. 41:463-474, 1971.

10. Kalmanson, G. M., Guze, L. B. Pyelonephritis. An attempt to demonstrate anti-kidney antibody in the sera of patients with chronic bacteriuria. Am. J. Med. Sci. 246:532-536, 1963.

11. Miller, T. E., Smith, J. W., Lehmann, J. W., Sanford, J. P. Autoimmunity in chronic experimental pyelonephritis. J. Infect. Dis. 122:191-195, 1970.

12. Coles, G. A., Chick, S., Hopkins, M., Ling, R., Radford, $\mathrm{N}$. J. The role of the $T$ cell in experimental pyelonephritis. Clin. Exp. Immunol. 16:629-636, 1974.

13. Pitchon, H. E., Kalmanson, G. M., Glassock, R. J., Ishida, K., Guze, L. B. Experimental enterococcal pyelonephritis in athymic (nude) mice [abstract no. 1070]. In Proceedings of the 19th Interscience Conference on Antimicrobial Agents and Chemotherapy. American Society for Microbiology, Washington, D.C., 1980.

14. Brooks, S. J. D., Lyons, J. M., Braude, A. I. Immunization against retrograde pyelonephritis. I. Production of an experimental model of severe ascending Escherichia coli pyelonephritis without bacteremia in rats. Am. J. Pathol. 74:345-358, 1974.

15. Brooks, S. J. D., Lyons, J. M., Braude, A. I. Immunization against retrograde pyelonephritis. III. Vaccination against chronic pyelonephritis due to Escherichia coli. J. Infect. Dis. 136:633-639, 1977.

16. Miller, T., Phillips, S. Pyelonephritis: the relationship between infection, renal scarring, and antimicrobial therapy. Kidney Int. 19:654-662, 1981.

17. Westenfelder, M., Galanos, C. Experimental lipid A-induced nephritis in the dog. A possible role of lipid $\mathrm{A}$ in the pathogenesis of abacterial chronic pyelonephritis. Infection 2:174-177, 1974.

18. Weissmann, G., Smolen, J. E., Korchak, H. M. Release of inflammatory mediators from stimulated neutrophils. N. Engl. J. Med. 303:27-34, 1980.

19. Babior, B. M. Oxygen-dependent microbial killing by phagocytes [in two parts]. N. Engl. J. Med. 298:659$668,721-725,1978$.

20. Maclean, D., Fishbein, M. C., Braunwald, E., Maroko, P. R. Long-term preservation of ischemic myocardium after experimental coronary artery occlusion. J. Clin. Invest. 61:541-551, 1978.

21. Seegmiller, J. E., Howell, R. R., Malawista, S. E. The inflammatory reaction to sodium urate: its possible relationship to the genesis of acute gouty arthritis. J.A.M.A. 180:469-475, 1962.

22. Malawista, S. E. Colchicine: a common mechanism for its anti-inflammatory and anti-mitotic effects. Arthritis Rheum. 11:191-197, 1968.

23. Malawista, S. E., Andriole, V. T. Colchicine: anti-inflammatory effect of low doses in a sensitive bacterial system. J. Lab. Clin. Med. 72:933-942, 1968. 
24. Sullivan, M. J., Harvey, R. A., Shimamura, T. The effects of cobra venom factor, an inhibitor of the complement system, on the sequence of morphological events in the rat kidney in experimental pyelonephritis. Yale J. Biol. Med. 50:267-273, 1977.

25. Shimamura, T. Mechanisms of renal tissue destruction in an experimental acute pyelonephritis. Exp. Mol. Pathol. 34:34-42, 1981.

26. Miller, T. E., Burnham, S., North, J. D. K. Immuno- logical enhancement in the pathogenesis of pyelonephritis. Clin. Exp. Immunol. 24:336-345, 1976.

27. Turk, J. L., Parker, D. The effect of cyclophosphamide on the immune response. J. Immunopharmacol. 1:127137, 1979.

28. Miller, T., Burnham, S., Simpson, G. Selective deficiency of thymus-derived lymphocytes in experimental pyelonephritis. Kidney Int. 8:88-97, 1975. 